\title{
Physical content of Heisenberg's uncertainty relation: Limitation and reformulation
}

\author{
Masanao Ozawa \\ Graduate School of Information Sciences, Tôhoku University, Aoba-ku, Sendai, 980-8579, Japan
}

\begin{abstract}
Heisenberg's reciprocal relation between position measurement error and momentum disturbance is rigorously proven under the assumption that those error and disturbance are independent of the state of the measured object. A generalization of Heisenberg's relation proven valid for arbitrary measurements is proposed and reveals two distinct types of possible violations of Heisenberg's relation.
\end{abstract}

PACS numbers: 03.65.Ta, 04.80.Nn, 03.67.-a

\section{INTRODUCTION}

An essential departure of quantum mechanics from classical mechanics is that any measurement of a microscopic object involves the interaction with the apparatus not to be neglected and accordingly introduces an unavoidable and uncontrollable disturbance on the measured object. Undoubtedly, this point of view led to fundamental doctrines of the Copenhagen interpretation of quantum mechanics [1], which successfully dissolved the wave-particle duality and the continuous-discontinuous discrepancy. In his celebrated paper 2] published in 1927, Heisenberg attempted to establish the quantitative expression of the amount of unavoidable momentum disturbance caused by any position measurement. His statement, with some elaborations, can be formulated as follows: For every measurement of the position $Q$ of a mass with root-mean-square error $\epsilon(Q)$, the root-meansquare disturbance $\eta(P)$ of the momentum $P$ of the mass caused by the interaction of this measurement always satisfies the relation

$$
\epsilon(Q) \eta(P) \geq \frac{\hbar}{2}
$$

Heisenberg 2] not only explained the physical intuition underlying the above relation by discussing the $\gamma$ ray microscope thought experiment, but also claimed that this relation can be proven as a straightforward consequence of the canonical commutation relation (CCR), $Q P-P Q=i \hbar$. A mathematical part of his proof was refined by introducing the notion of standard deviation shortly afterward by Kennard [3] and later generalized to arbitrary pair of observables by Robertson [4] as the following statement: For any pair of observables $A$ and $B$, their standard deviations, $\sigma(A)$ and $\sigma(B)$, satisfy the relation

$$
\sigma(A) \sigma(B) \geq \frac{1}{2}|\langle\psi,[A, B] \psi\rangle|
$$

in any state $\psi$ with $\sigma(A), \sigma(B)<\infty$. In the above, $[A, B]$ stands for the commutator $[A, B]=A B-B A$, and the standard deviation is defined as $\sigma(A)=\left(\left\langle\psi, A^{2} \psi\right\rangle-\right.$ $\left.\langle\psi, A \psi\rangle^{2}\right)^{1 / 2}$, where $\langle\cdots, \cdots\rangle$ denotes the inner product 5]. As a consequence of $[Q, P]=i \hbar$, we have

$$
\sigma(Q) \sigma(P) \geq \frac{\hbar}{2}
$$

which was proven by Heisenberg himself for Gaussian states and by Kennard [3] generally.

Heisenberg 2] argued that the mathematical relation Eq. (3) concludes the physical assertion expressed by Eq. (11). Since then, his claim has been accepted by many $6,7,8,9,10,11,12$. However, the universal validity of Eq. (1) has been also criticized in many ways 13, 14, 15, $16,17,18,19,20,21,22,23,24,[25,[26$, 27, [28, 29]. In fact, the "resolution power" of the $\gamma$ ray microscope cannot be identified in any interpretation with the standard deviation of the mass position in the state to be measured. Thus, Eq. (3) cannot be considered as a "formal expression" of Eq. (1). Moreover, no one has succeeded in proving Eq. (11) for general measurements even by applying Eq. (3) not only to the mass state but also to the apparatus state. Undoubtedly, this has caused serious confusions among physicists on the status of this leading principle of quantum mechanics.

In order to clarify the confusion, we shall start with precise definitions of root-mean-square disturbance and mean-square error in arbitrary measurements and reconstruct Heisenberg's argument. His argument applies Eq. (3) to the mass state just after the measurement. However, in order to derive Eq. (1), additional assumptions are necessary. Here, we shall show that the following two assumptions completes Heisenberg's argument: (i) Both amounts of $\epsilon(Q)$ and $\eta(P)$ are independent of the input state. (ii) The measurement always leaves the mass with position standard deviation smaller than the $\epsilon(Q)$. Thus, Heisenberg's argument left open the following questions: Can we further relax the assumptions? What relation holds for arbitrary measurements? What conditions characterize violations of Eq. (11)? In particular, the second assumption, which will be called the equipredictivity of measurement, stringently restricts the class of measurements to which Eq. (11) is applicable. In order to answer those questions, a new approach to proving Eq. (11) will be proposed based on analysis of commutation relations for the operators representing the error and the disturbance. From this approach, we shall obtain a generalization of Heisenberg's relation proven valid for arbitrary measurements and also obtained a new proof of Heisenberg's relation without assuming the equipredictivity. We shall further discuss limitations on Heisenberg's relation based on the generalized relation and show 
that there are two distinct types of measurements in which Heisenberg's relation is violated uniformly for any input state. The significance of the results will be discussed in the final section. In this Letter, we shall confine our attention to the position measurement error and momentum disturbance; generalizations to arbitrary observables are partly discussed in a separate paper [30].

\section{FORMULATION OF ERROR AND DISTURBANCE}

Let us consider a measurement of position $Q$ of a mass with momentum $P$. The interaction between the mass and the apparatus is assumed to turn on at time 0 and turn off at time $\Delta t$. Let $\psi$ be the input state, i.e., the state of the mass just before the interaction, and let $\xi$ be the state of the apparatus just before the interaction. Let $U$ be the unitary operator representing the time evolution of the mass plus apparatus for the time interval $(0, \Delta t)$. Then, in the Heisenberg picture the momentum change is represented by

$$
D(P)=P(\Delta t)-P(0),
$$

where $P(0)=P \otimes I$ and $P(\Delta t)=U^{\dagger} P(0) U$. The rootmean-square momentum disturbance is defined by

$$
\eta(P)=\left\langle D(P)^{2}\right\rangle^{1 / 2},
$$

where $\langle\cdots\rangle$ denotes the mean value in the original state $\psi \otimes \xi$.

Now we shall discuss some basic properties of the above quantity. The above statistical definition leads to the following geometric expression

$$
\eta(P)=\|P(\Delta t) \psi \otimes \xi-P(0) \psi \otimes \xi\| .
$$

Thus, by the relation

$$
\sigma(A)=\|A \psi \otimes \xi-\langle A\rangle \psi \otimes \xi\|
$$

for any observable $A$, we easily obtain the following relation

$$
|\sigma(P(\Delta t))-\sigma(P(0))| \leq \eta(P)+|\langle P(\Delta t)\rangle-\langle P(0)\rangle|,
$$

stating that the change in the momentum standard deviation is bounded from above by the root-mean-square disturbance except for the change in the mean momentum. If the mass had a definite momentum $p$ before the measurement, i.e., $P(0) \psi=p \psi$, we would have

$$
D(P) \psi \otimes \xi=[P(\Delta t)-p] \psi \otimes \xi,
$$

and hence

$$
\eta(P)^{2}=\left\langle[P(\Delta t)-p]^{2}\right\rangle \geq \sigma(P(\Delta t))^{2} .
$$

Thus, the momentum standard deviation after the measurement is bounded from above by the root-mean-square disturbance. In particular, if $p=0$, we have

$$
\eta(P)^{2}=\left\langle P(\Delta t)^{2}\right\rangle .
$$

This relation can be interpreted as follows. If the mass is at rest before the measurement, all the object's momentum after the measurement arises from the interaction, so that the mean-square disturbance (i.e., the square of the root-mean-square disturbance) is equal to the meansquare momentum after the measurement.

The role of the interaction is to transduce the value of $Q$ before the interaction to the value of an observable $M$ of the probe, a part of the apparatus, after the interaction. We shall call $M$ the meter observable. We suppose that after the interaction is turned off, the outcome of the $Q$ measurement in the state $\psi$ is obtained by measuring $M$ without further disturbing the momentum $P$ of the mass; this is possible by another measuring apparatus coupled only to the probe. The postulates of quantum mechanics do not limit the accuracy of the latter measurement of $M$, and hence we neglect the error from this measurement. Then, in the Heisenberg picture the error caused by this process of the $Q$ measurement is represented by

$$
N(Q)=M(\Delta t)-Q(0),
$$

where $Q(0)=Q \otimes I$ and $M(\Delta t)=U^{\dagger}(I \otimes M) U$. The root-mean-square error is defined by

$$
\epsilon(Q)=\left\langle N(Q)^{2}\right\rangle^{1 / 2} .
$$

The above statistical definition leads to the following geometric expression

$$
\epsilon(Q)=\|M(\Delta t) \psi \otimes \xi-Q(0) \psi \otimes \xi\| .
$$

Analogously with Eq. (15), we obtain

$$
|\sigma(M(\Delta t))-\sigma(Q(0))| \leq \epsilon(Q)+|\langle M(\Delta t)\rangle-\langle Q(0)\rangle|,
$$

showing that the change in the standard deviation from input to output is bounded from above by the root-meansquare noise except for the bias, namely, the change in the mean value. If the mass had a definite position $q$ before the measurement, we would have

$$
\epsilon(Q)^{2}=\left\langle[M(\Delta t)-q]^{2}\right\rangle \geq \sigma(M(\Delta t))^{2},
$$

so that $\epsilon(Q)$ would represent the root-mean-square deviation of the measurement outcome from the true position $q$ and would limit the standard deviation of the outcome of the $Q$ measurement.

\section{RECONSTRUCTION OF HEISENBERG'S ARGUMENT}

In the 1927 paper, Heisenberg claimed that Eq. (11) is a straightforward mathematical consequence of the CCR, $Q P-P Q=i \hbar$. Although he gave a claimed proof of Eq. (11) from the CCR, his argument includes some implicit assumptions. Consequently, Eq. (11) cannot be considered as a straightforward consequence of the CCR nor 
as a universally valid relation. Heisenberg's idea of the proof is as follows: Start with a measurement with error $\epsilon(Q)$, claim that the state just after the measurement has $\sigma(Q)=\epsilon(Q)$, prove Eq. (3) from the CCR for the above state to obtain the relation $\epsilon(Q) \sigma(P) \geq \hbar / 2$, and identify $\sigma(P)$ with $\eta(P)$ to obtain Eq. (11). In order to clarify the hidden assumptions, in what follows, we shall show that Heisenberg's original proof can be rigorously reconstructed under the following two additional assumptions: (i) Both $\epsilon(Q)$ and $\eta(P)$ are independent of the input state; in this case, we say that the measurement has constant mean-square noise and disturbance. (ii) The measurement always leaves the mass with position standard deviation smaller than $\epsilon(Q)$; in this case, we say that the measurement is equipredictive.

Under the above assumptions, the proof of Eq. (1) runs as follows. In order to obtain an estimate of the momentum disturbance, we shall consider the case where the mass were at rest before the measurement. Then, by Eq. (11) the object's mean-square momentum after the measurement is equal to the mean-square momentum disturbance, i.e.,

$$
\eta(P)^{2}=\left\langle P(\Delta t)^{2}\right\rangle
$$

Let $\psi_{x}$ be the state of the mass after the measurement with outcome $x$. We shall denote by $\sigma_{x}$ the standard deviation in the state $\psi_{x}$. We shall later show that the relation

$$
\eta(P) \geq \sigma_{x}(P)
$$

holds with positive probability. On the other hand, by condition (ii), we have

$$
\epsilon(Q) \geq \sigma_{x}(Q)
$$

holds with probability one. It follows from Eqs. (18) and (19) that there must be a state vector $\psi_{x}$ satisfying

$$
\epsilon(Q) \eta(P) \geq \sigma_{x}(Q) \sigma_{x}(P) .
$$

Thus, Eq. (11) follows from Eq. (3), if the mass is at rest just before the measurement. Then, assumption (i) ensures that Eq. (1) holds irrespective of the particular choice of the input state. We, therefore, conclude that every equipredictive measurement with constant meansquare noise and disturbance satisfies Eq. (1) for every input state [31].

Now, we shall prove Eq. (18). The state $\psi_{x}$ arises with the probability distribution $\pi(d x)$ of obtaining the outcome $x$ determined by

$$
\pi(d x)=\left\langle E^{M(\Delta t)}(d x)\right\rangle
$$

where $E^{M(\Delta t)}$ is the resolution of the identity corresponding to the observable $M(\Delta t)$. Then, since $\left\langle P(\Delta t)^{2}\right\rangle$ is the mean of $P^{2}$ after the measurement, we have

$$
\left\langle P(\Delta t)^{2}\right\rangle=\int\left\langle\psi_{x}, P^{2} \psi_{x}\right\rangle \pi(d x)
$$

Since $\left\langle\psi_{x}, P^{2} \psi_{x}\right\rangle \geq \sigma_{x}(P)^{2}$ for any $x$, we have

$$
\int\left\langle\psi_{x}, P^{2} \psi_{x}\right\rangle \pi(d x) \geq \int \sigma_{x}(P)^{2} \pi(d x),
$$

and consequently

$$
\left\langle P(\Delta t)^{2}\right\rangle \geq \int \sigma_{x}(P)^{2} \pi(d x)
$$

Thus, we have

$$
\left\langle P(\Delta t)^{2}\right\rangle \geq \sigma_{x}(P)^{2},
$$

with positive probability; otherwise the opposite inequality of Eq. (24) would hold. This concludes the proof of Eq. (18).

\section{UNIVERSALLY VALID REFORMULATION}

The above proof of Eq. (1) rigorously reproduces Heisenberg's intention in 1927 that the uncertainty relation expressed by Eq. (1) is a straightforward consequence from Eq. (3) for the state after the measurement. However, the above proof does not show that Eq. (11) is universally valid and has left the problem quite open as to the limitation of Eq. (11). Thus, the following problems have remained open on Heisenberg's uncertainty relation. Can we further relax the assumptions of the proof? What relation holds for arbitrary measurements? What conditions characterize violations of Eq. (1)?

In what follows, we shall prove that the relation

$$
\epsilon(Q) \eta(P)+\epsilon(Q) \sigma(P)+\sigma(Q) \eta(P) \geq \frac{\hbar}{2}
$$

holds for every measurement and every input state as long as all the relevant terms are finite, where $\sigma(Q)$ and $\sigma(P)$ refer to the standard deviations of $Q$ and $P$ in the input state.

The proof of Eq. (26) runs as follows. Since $M$ and $P$ are observables in different systems, we have $[M(\Delta t), P(\Delta t)]=0$. Substituting $M(\Delta t)=Q(0)+$ $N(Q)$ and $P(\Delta t)=P(0)+D(P)$ for this relation and using the commutation relation $[Q, P]=i \hbar$, we have [18, 23, 24]

$$
[N(Q), D(P)]+[N(Q), P(0)]+[Q(0), D(P)]=-i \hbar .
$$

Taking the moduli of means of the both sides and applying the triangular inequality, we have

$$
\begin{aligned}
|\langle[N(Q), D(P)]\rangle|+|\langle[N(Q), P(0)]\rangle| & \\
+ & |\langle[Q(0), D(P)]\rangle| \geq \hbar
\end{aligned}
$$

Since the variance is not greater than the mean-square, Eq. (2) gives

$$
\epsilon(Q) \eta(P) \geq \sigma[N(Q)] \sigma[D(P)] \geq \frac{1}{2}|\langle[N(Q), D(P)]\rangle|,
$$


where $\sigma$ in the middle term refers to the standard deviation in the state $\psi \otimes \xi$. Similarly, we have

$$
\begin{aligned}
\epsilon(Q) \sigma(P) & \geq \sigma[N(Q)] \sigma[P(0)] \geq \frac{1}{2}|\langle[N(Q), D(P)]\rangle|, \\
\sigma(Q) \eta(P) & \geq \sigma[Q(0)] \sigma[D(P)] \geq \frac{1}{2}|\langle[Q(0), D(P)]\rangle| .
\end{aligned}
$$

Thus, from Eqs. (28)- 31), we conclude Eq. (26).

\section{LIMITATION OF HEISENBERG'S RELATION}

The above proof clearly answers the question when Eq. (11) holds generally: If $[N(Q), P(0)]+[Q(0), D(P)]=$ 0, then Eq. (11) holds for every input state $\psi$ and every apparatus state $\xi$. In fact, from Eq. (27), the condition $[N(Q), P(0)]+[Q(0), D(P)]=0$ implies the commutation relation

$$
[N(Q), D(P)]=-i \hbar .
$$

Thus, Eq. (11) follows immediately from Eq. (29).

In order to draw a useful conclusion, we say that the measurement has independent intervention, if there are two operators $N$ and $D$ on the state space of the apparatus such that

$$
\begin{aligned}
& N(Q)=I \otimes N, \\
& D(P)=I \otimes D, .
\end{aligned}
$$

In this case, we have $\epsilon(Q)=\|N \xi\|$ and $\eta(P)=\|D \xi\|$, so that every measurement with independent intervention has constant mean-square noise and disturbance.

From Eqs. (33) and (34) the relations

$$
[N(Q), P(0)]=[Q(0), D(P)]=0
$$

follows easily. Thus, we conclude that every measurement with independent intervention satisfies Eq. (1) for every input state $\psi$ and every apparatus state $\xi$ without requiring the equipredictivity.

The above conclusion suggests that the equipredictivity is not an essential requirement for a measurement to satisfy Heisenberg's relation compared with the independence of the noise and the disturbance from the measured system.

\section{VON NEUMANN MODELS}

Von Neumann [6] constructed the first quantum mechanical model of position measurement. His model is described by the Hamiltonian

$$
H=K\left(Q \otimes P_{0}\right)
$$

that couples the mass position $Q$ and the probe momentum $P_{0}$ with coupling constant $K$ satisfying $K \Delta t=1$.
Then, taking the meter to be the probe position, i.e., $M=Q_{0}$, the time evolution $U=\exp (-i \Delta t H / \hbar)$ determines the input-output relation 29]

$$
\begin{aligned}
M(\Delta t) & =Q(0)+\left(I \otimes Q_{0}\right), \\
P(\Delta t) & =P(0)-\left(I \otimes P_{0}\right) .
\end{aligned}
$$

Thus, this model has independent intervention with $N(Q)=I \otimes Q_{0}$ and $D(P)=-I \otimes P_{0}$, and hence Eq. (10) holds. Assuming that the probe's mean position is initially at the origin, the constant root-mean-square error $\epsilon(Q)=\sigma\left(Q_{0}\right)$ plays an analogous role of "resolution power" of microscopic measurements. Then, Eq. (11) follows directly from the relation $\sigma\left(Q_{0}\right) \sigma\left(P_{0}\right) \geq \hbar / 2$ [29].

\section{EQUIVALENCE OF MEASUREMENTS WITH INDEPENDENT INTERVENTION}

Interestingly, we can show that every measurement with independent intervention has the position error operator and the momentum disturbance operator equivalent to those of the above von Neumann model. In fact, from Eqs. (32), (33), and (34), we have

$$
[N,-D]=i \hbar .
$$

Thus, by the Stone-von Neumann theorem on the uniqueness of representations of the canonical commutation relations [32], the independent error and disturbance operators can be, up to unitary equivalence, decomposed as

$$
\begin{aligned}
N & =Q_{0} \otimes I, \\
-D & =P_{0} \otimes I,
\end{aligned}
$$

for a one-dimensional position $Q_{0}$ and momentum $P_{0}$. Then, the input-output relation can be, up to local unitary equivalence, represented as

$$
\begin{aligned}
M(\Delta t) & =Q(0)+\left(I \otimes Q_{0} \otimes I\right), \\
P(\Delta t) & =P(0)-\left(I \otimes P_{0} \otimes I\right) .
\end{aligned}
$$

Thus, every measurement with independent intervention has the position error and momentum disturbance equivalent with the von Neumann model.

\section{VIOLATIONS OF HEISENBERG'S RELATION}

For finitely accessible input states, i.e., $\sigma(Q), \sigma(P)<$ $\infty$, Eq. 26) excludes the possibility of having both $\epsilon(Q)=0$ and $\eta(P)=0$ simultaneously. However, it is possible to have $\eta(P)=0$ uniformly over every input state or alternatively to have $\epsilon(Q)=0$ uniformly. In both cases, Eq. (1) is violated uniformly with $\epsilon(Q) \eta(P)=0$. Thus, we have two types of uniform violations of Eq. (1); 
we shall refer to the former as type $I$ and the latter as type II.

In type I violations, by substituting $\eta(P)=0$ in Eq. (26), we have

$$
\epsilon(Q) \sigma(P) \geq \frac{\hbar}{2}
$$

The above relation even allows to have $\epsilon(Q) \rightarrow 0$ with $\sigma(P) \rightarrow \infty$, and actually a model in Section IX will realize relations $\eta(P)=0$ and $\epsilon(Q) \rightarrow 0$ with $\sigma(P) \rightarrow \infty$. In this case, the small error is compensated not by the large momentum disturbance but by the large initial momentum uncertainty. From Eq. (3), this means that without disturbing the momentum the position can be measured as precisely as our initial knowledge on the object position. This rather natural possibility has been excluded from Eq. (1).

Similarly, in type II violations, $\sigma(Q)$ and $\eta(P)$ are constrained as

$$
\sigma(Q) \eta(P) \geq \frac{\hbar}{2}
$$

so that the small momentum disturbance is compensated by the large initial position uncertainty, and actually a model in Ref. 29] realizes relations $\epsilon(Q)=0$ and $\eta(P) \rightarrow 0$ with $\sigma(Q) \rightarrow \infty$. From Eq. (3), this implies the possibility of the precise position measurement with only disturbing the momentum as much as the initial momentum uncertainty. Since Eq. (1) has prohibited the precise position measurement without infinite momentum transfer, this opens a new possibility of precision measurements of the mass position and similar physical quantities.

\section{TYPE I VIOLATIONS AND EPR THOUGHT EXPERIMENTS}

A Type I violation can be obtained by reformulating the Einstein-Podolsky-Rosen thought experiment [13]. Let the measured system be a two-particle system comprising one-dimensional particles 1 and 2 with positions $Q_{1}, Q_{2}$ and momenta $P_{1}, P_{2}$, respectively, and consider the following process of measuring $Q_{1}$; our measuring apparatus is assumed to couple only to particle 2 and to precisely measure $Q_{2}$, but then to output this measured value of $Q_{2}$ as the outcome of the indirect $Q_{1}$ measurement. This is generally not a good measurement of $Q_{1}$; however, the interaction for this measurement does not disturb $P_{1}$, so that $\eta\left(P_{1}\right)=0$ uniformly. In this case, we can show that $\epsilon\left(Q_{1}\right)^{2}=\left\langle\left(Q_{1}-Q_{2}\right)^{2}\right\rangle$ for any input state 33]. On the other hand, for any small $\alpha>0$, we can choose a two-particle state $\psi$ such that $\left\langle\left(Q_{1}-Q_{2}\right)^{2}\right\rangle<\alpha^{2}$. Thus, in all such states, we can measure $Q_{1}$ with $\epsilon\left(Q_{1}\right)<\alpha$ without disturbing $P_{1}$.

The importance of the above example is the abundance of such state $\psi$. Let $\mathcal{H}_{i}$ be the state space of particle $i$ for $i=1,2$. There exist a unitary operator $U$ on the space
$\mathcal{H}_{1} \otimes \mathcal{H}_{2}$ and a state $\eta^{\prime} \in \mathcal{H}_{2}$ such that for every $\eta$ the state $\psi=U\left(\eta \otimes \eta^{\prime}\right)$ satisfies the condition $\left\langle\left(Q_{1}-Q_{2}\right)^{2}\right\rangle<$ $\alpha^{2}$.

\section{TYPE II VIOLATIONS}

All the type II violations are characterized by the condition $N(Q)=0$. Thus, the input-output relation of such measurement is characterized by

$$
\begin{aligned}
M(\Delta t) & =Q(0) \\
P(\Delta t) & =P(0)+D(P) .
\end{aligned}
$$

It is generally accepted that every measurement is associated with a probability operator-valued measure (POVM) $\Pi$, which maps every interval $\Delta$ to a positive operator $\Pi(\Delta)$ on the state space of the measured object 34, 35]; the POVM determines the probability of obtaining the outcome $\mathbf{x}$ of the measurement in an interval $\Delta$ on input state $\psi$ by $\operatorname{Pr}\{\mathbf{x} \in \Delta\}=\langle\psi, \Pi(\Delta) \psi\rangle$. Then, by Eq. (46) the measurement is of type II violation if and only if for any interval $\Delta$ the operator $\Pi(\Delta)$ is the spectral projection of $Q$ corresponding to $\Delta$. All the possible state changes associated with those measurements of type II violation were described in Ref. 36.

A model measurement of type II violation arose from the controversy on the sensitivity limit of gravitational wave detectors (see [37] for a brief survey). The sensitivity limit of interferometer type gravitational wave detectors is derived from the accuracy of the monitoring of a free mass position, the relative position of mirrors in the interferometer. Braginsky, Caves, and others 10, 11, 25. claimed that any position measurement results a backaction obeying Eq. (11) that leads to the following sensitivity limit, called the standard quantum limit (SQL), for monitoring the free-mass position: Let a free mass $m$ undergo unitary evolution during the time $\tau$ between two measurements of its position $Q$, made with identical measuring apparatuses; the result of the second measurement cannot be predicted with root-mean-square error smaller than $(\hbar \tau / m)^{1 / 2}$ in average [38]. However, Yuen 16. claimed that the SQL is not universally valid and can be breached by a "contractive state measurement" that leaves the free-mass in a state with decreasing position uncertainty in time. After the debate on the realizability of contractive state measurements, it was shown in Ref. 19] that the contractive state measurement can be realized by the interaction

$$
H=\frac{K \pi}{3 \sqrt{3}}\left\{2\left(Q \otimes P_{0}-P \otimes Q_{0}\right)+\left(Q P \otimes I-I \otimes Q_{0} P_{0}\right)\right\},
$$

where $Q_{0}$ and $P_{0}$ are the probe position and probe momentum, respectively. We can show that this measurement not only breaks the SQL [19] but also is a type II violation of Heisenberg's relation. In fact, with the meter observable $M=Q_{0}$, the time evolution $U=$ 
$\exp (-i \Delta t H / \hbar)$ with $K \Delta t=1$ determines the inputoutput relation 29]

$$
\begin{aligned}
M(\Delta t) & =Q(0), \\
P(\Delta t) & =P(0)+\left[P_{0}(0)-P(0)\right] .
\end{aligned}
$$

From the above input-output relation, we have $N(Q)=0$ and $D(P)=P_{0}(0)-P(0)$, so that $\epsilon(Q)=0$ uniformly and $\eta(P)^{2}=\left\langle P_{0}(0)^{2}\right\rangle+\left\langle P(0)^{2}\right\rangle-2\left\langle P_{0}(0)\right\rangle\langle P(0)\rangle<\infty$. Thus, $\epsilon(Q) \eta(P)=0$ uniformly and the above measurement is of type II violation of Eq. (1). For more detail on this measurement, we refer to Refs. 19, 20, 28, 29, 30, 39.

\section{CONCLUDING REMARKS}

Many text books have associated the formal expression of "Heisenberg's uncertainty relation" to Eq. (3), but also associated the physical meaning of "Heisenberg's uncertainty relation" to Eq. (11). However, Eq. (3) can no longer be considered as a formal expression of Eq. (1), since Eq. (3) is a universally valid mathematical relation, but Eq. (11) has its own limitation.

In this Letter, we define "Heisenberg's uncertainty relation" based on Heisenberg's claim published in 1927, which, of course, revealed the limitation of our ability of observation on microscopic objects at the first time. However, Heisenberg himself appears to have changed his position from 1927 to 1929. Around this time, it was already known that the EPR type thought experiment violates Eq. (1). In this case, the position of the mass at a time $t$ can be indirectly measured very precisely without disturbing the momentum, and hence if the momentum is measured directly just after the position measurement, the momentum at the time $t$ can also be measured very precisely, as discussed in Section [IX] Heisenberg's response to this criticism appears to have been that he considered the uncertainty relation to be Eq. (3) rather than Eq. (11) by stating, for instance, that "every experiment destroys some of the knowledge of the system which was obtained by previous experiments. This formulation makes it clear that the uncertainty relation does not refer to the past" (p. 20, Ref. [40]). Heisenberg's response means that even if we can measure both the position and the momentum at the past time $t$ very precisely, after the momentum measurement the mass has no longer definite position so that Eq. (3) is not violated at any time. Thus, around this time, "Heisenberg's uncertainty relation" might turn to be a more fundamental formal relation like the CCR than what Heisenberg claimed in 1927.

Although there have been many attempts to show violations of Eq. (1), it has been difficult to construct a forceful argument without definite definitions of error and disturbance, since, as shown in Section VIII any such conceivable violation has an alternative compensating reciprocal relation, Eq. (44) or Eq. (45), with the same lower bound. For instance, if one shows a calculation on some model indicating that Eq. (1) is ultimately violated, then the defender can easily modify the calculation to lead to a reciprocal relation Eq. (44) or Eq. (45) and tell that not only Eq. (3) but also "Heisenberg's uncertainty relation" holds in this measurement.

Thus, the definiteness of the definition of error is quite important in the whole story. We can justify our definition of error as follows. If the input state has the true value of the measured observable, our definition gives the root-mean-square difference between the true value and the measured value, and hence this is the only one from the name "root-mean-square error." We can also show that under some natural mathematical requirements, extending the definition to arbitrary input states is also unique. In this Letter, the root-mean-square error is defined through the model of measuring process. However, this error can be shown to be model independent and actually determined directly from the POVM of the measurement. In fact, the error $\epsilon(A)$ in measuring an observable $A$ using apparatus with a POVM $\Pi(d x)$ coincides with the distance $d(\Pi, A)$ between the POVM $\Pi(d x)$ and the observable $A$ defined by

$$
\begin{aligned}
d(\Pi, A)^{2}= & \left\langle\psi\left|\int x^{2} \Pi(d x)-\left[\int x \Pi(d x)\right]^{2}\right| \psi\right\rangle \\
& +\left\|\int x \Pi(d x) \psi-A \psi\right\|^{2} .
\end{aligned}
$$

The detail will be discussed in a forthcoming paper [41]. According to that, the definition of root-mean-square error is believed to be unique.

The $\gamma$ ray microscope is considered to be described by a model with independent intervention under usual approximation allowed in mathematical modeling of the physical apparatus. Then, the theory shows that the root-mean-square error and the root-mean-square momentum disturbance satisfy Heisenberg's relation. The resolution power of the $\gamma$ ray microscope is considered to coincide with the experimental root-mean-square error in the state with definite position before measurement up to constant factor. Thus, the resolution power should coincide with the root-mean-square error of the model up to constant factor, so that the theory consistently concludes that the product of the resolution power and the momentum disturbance is lower bounded by the Planck constant times a constant factor.

In the present Letter, we have shown that Heisenberg's reciprocal relation between position-measurement error and momentum disturbance holds for every measurement with independent intervention and clarifies the limitation of Heisenberg's relation. We also proposed a new universally valid relation among measurement error, disturbance, and initial uncertainties. This relation reveals possibilities of measurements beyond Heisenberg's relation and clarifies the new constraints for measurements beyond Heisenberg's relation. 


\section{Acknowledgments}

This work was supported by the Strategic Information and Communications R\&D Promotion Scheme of the
MPHPT of Japan, by the CREST project of the JST, and by the Grant-in-Aid for Scientific Research of the JSPS.
[1] N. Bohr, Nature (London) 121, 580 (1928).

[2] W. Heisenberg, Z. Phys. 43, 172 (1927) [in Quantum Theory and Measurement, edited by J. A. Wheeler and W. H. Zurek, pages 62-84 (Princeton University Press, 1983)].

[3] E. H. Kennard, Z. Physik 44, 326 (1927).

[4] H. P. Robertson, Phys. Rev. 34, 163 (1929).

[5] In this Letter, every state vector is assumed normalized and the domain of the commutator $[A, B]$ is considered extended appropriately.

[6] J. von Neumann, Mathematische Grundlagen der Quantenmechanik (Springer, Berlin, 1932) [Mathematical Foundations of Quantum Mechanics (Princeton University Press, 1955)].

[7] N. Bohr, in Albert Einstein: Philosopher-Scientist, edited by P. A. Shilpp, pages 200-241 (Evanston, The Library of Living Philosophers, 1949).

[8] D. Bohm, Quantum Theory (Prentice-Hall, New York, 1951).

[9] A. Messiah, Mécanique Quantique, I (Dunod, Paris, 1959), [Quantum Mechanics, I (North-Holland, Amsterdam, 1959)].

[10] V. B. Braginsky and Yu. I. Vorontsov, Uspehi Fiz. Nauk 114, 41 (1974) [Sov. Phys. Usp. 17, 644 (1975)].

[11] C. M. Caves et al., Rev. Mod. Phys. 52, 341 (1980).

[12] V. B. Braginsky et al., Science 209, 547 (1980).

[13] A. Einstein, B. Podolsky, and N. Rosen, Phys. Rev. 47, 777 (1935).

[14] E. Arthurs and J. L. Kelly, Jr., Bell. Syst. Tech. J. 44, 725 (1965).

[15] L. E. Ballentine, Rev. Mod. Phys. 42, 358 (1970).

[16] H. P. Yuen, Phys. Rev. Lett. 51, 719 (1983).

[17] K. Kraus. Phys. Rev. D 35, 3070 (1987).

[18] E. Arthurs and M. S. Goodman, Phys. Rev. Lett. 60, 2447 (1988).

[19] M. Ozawa, Phys. Rev. Lett. 60, 385 (1988).

[20] M. Ozawa, in Squeezed and Nonclassical Light, edited by P. Tombesi and E. R. Pike, pages 263-286 (Plenum, New York, 1989).

[21] J. Hilgevoord and J. Uffink, in Sixty-Two Years of Uncertainty, edited by A. I. Miller, pages 121-137 (Plenum, New York, 1990).

[22] H. Martens and W. M. de Muynck, Found. Phys. 20, 357
(1990).

[23] M. Ozawa, in Quantum Aspects of Optical Communications, edited by C. Bendjaballah et al., pages 3-17 (Springer, Berlin, 1991).

[24] S. Ishikawa, Rep. Math. Phys. 29, 257 (1991).

[25] V. B. Braginsky and F. Ya. Khalili, Quantum Measurement, (Cambridge University Press, Cambridge, 1992).

[26] H. Martens and W. M. de Muynck, J. Phys. A 25, 4887 (1992).

[27] D. M. Appleby, Int. J. Theor. Phys. 37, 1491 (1998).

[28] M. Ozawa, Phys. Lett. A 282, 336 (2001).

[29] M. Ozawa, Phys. Lett. A 299, 1 (2002).

[30] M. Ozawa, Phys. Rev. A 67, 042105 (2003).

[31] In the above proof, we illegitimately assume that the input state is a momentum eigenstate, and that the output state is a well-defined pure state. The argument can be improved so that the input state is assumed to be a normalized state arbitrarily close to the momentum eigenstate. Then, the output state is generally well-defined as a density operator. Mathematically complete argument along with this line is more involved and will be given elsewhere.

[32] M. Reed and B. Simon, Methods of Modern Mathematical Physics, II: Fourier Analysis, Self-Adjointness. (Academic, New York, 1975).

[33] Since $Q_{2}$ is measured precisely, we have $N\left(Q_{2}\right)=$ $M(\Delta t)-Q_{2}(0)=0$, so that $N\left(Q_{1}\right)=M(\Delta t)-Q_{1}(0)=$ $Q_{2}(0)-Q_{1}(0)$, and hence we have $\epsilon\left(Q_{1}\right)^{2}=\left\langle\left(Q_{1}-Q_{2}\right)^{2}\right\rangle$.

[34] E. B. Davies, Quantum Theory of Open Systems, (Academic, London, 1976).

[35] M. Ozawa, J. Math. Phys. 25, 79 (1984).

[36] M. Ozawa, in Quantum Communication, Computing, and Measurement 3, edited by P. Tombesi and O. Hirota, pages 97-106 (Kluwer/Plenum, New York, 2001).

[37] J. Maddox, Nature (London) 331, 559 (1988).

[38] C. M. Caves, Phys. Rev. Lett. 54, 2465 (1985).

[39] M. Ozawa, Phys. Rev. A 41, 1735 (1990).

[40] W. Heisenberg, The Physical Principles of the Quantum Theory, (University of Chicago Press, Chicago, 1930) [Reprinted by Dover, New York $(1949,1967)]$.

[41] M. Ozawa, in preparation. 